\title{
Interference on cytoplasmic polyadenylation element-binding proteins affects the invasion ability of glioma stem cells
}

\author{
H.L. Liu*, J.F. Huo*, Z.J. Liu and X.B. Chen \\ Department of Neurosurgery, Huaihe Hospital, Henan University, Kaifeng, China \\ *These authors contributed equally to this study. \\ Corresponding author: X.B. Chen \\ E-mail: chenxbdn@163.com
}

Genet. Mol. Res. 14 (4): 13504-13510 (2015)

Received March 3, 2015

Accepted June 18, 2015

Published October 28, 2015

DOI http://dx.doi.org/10.4238/2015.October.28.11

\begin{abstract}
Glioma stem cells derived from primary cultures were divided into an experiment group, a control group, and a blank group and subjected to cytoplasmic polyadenilation element-binding protein (CPEBs) interference, transfection with empty vector, and normal culture, respectively, to compare their invasion abilities. Western blotting showed that siRNA-3 had the strongest interfering effect on CPEBs. CPEBs were expressed in the experiment group with green fluorescence at an expression rate of over $70 \%$. Significantly lower CPEB expression was observed in the experiment group compared to in the control and blank groups $(P<0.05)$. After $48-h$ treatment, the apoptotic rate in the experiment group was $21.43 \%$, which was significantly higher than that in the blank $(0.51 \%)$ and control $(1.43 \%)$ groups $(P<0.05)$. After 3 days of treatment, the experiment group grew significantly more slowly than did the control and blank groups $(P<0.05)$. The transwell invasion assay showed that significantly fewer cells in the experiment group penetrated the membrane than did cells in the control and blank groups $(P<0.05)$. After CPEB interference, the growth, proliferation,
\end{abstract}


and invasion of glioma stem cells were substantially inhibited, providing support for targeted therapy of glioma and for improving prognosis.

Key words: Cytoplasmic polyadenylation element-binding protein; Glioma stem cell; Invasive capability

\section{INTRODUCTION}

Cytoplasmic polyadenylation element-binding proteins (CPEBs) play important roles in the development, differentiation, aging, and apoptosis of stem cells by affecting cytoplasmic polyadenylation through mediating mRNA translation (D'Ambrogio et al., 2013). Although the expression of CPEBs in various tumor tissues has been studied, few studies have examined targeted therapy (Bishnoi et al., 2008). In this study, we analyzed the effects of CPEB interference on the invasion ability of glioma stem cells (GSCs).

\section{MATERIAL AND METHODS}

\section{Main materials}

The U87 cell line was provided by the American Type Culture Collection (Manassas, VA, USA) and used for primary culture at room temperature in DMEM containing $10 \%$ fetal bovine serum to obtain GSCs. The pSRL-SIH1-H1-GFP plasmid (Tianjin Ssier Biotechnology Co., Ltd., Tianjin, China) was used as the lentiviral vector (Lee et al., 2013). Escherichia coli strain DH5a [TaKaRa Biotechnology (Dalian) Co., Ltd., Shiga, Japan] was used as competent cells (Xiao et al., 2008).

\section{Methods}

\section{Preparation of lentiviral vector}

siRNAs were synthesized by designing 3 target sequences for RNA interference based on the gene sequences of CPEBs to transfect U87 cells. The siRNA sequence with the most evident interfering effect on CPEBs was identified by western blotting according to which DNA oligo was prepared, with the sticky ends identical to the restriction sites of the pSRL-SIH1-H1-GFP plasmid (Lee et al., 2013). The products were then transfected into $E$. coli competent cells, and preparation of lentiviral vector was confirmed through cloning, extraction, digestion, identification, and sequencing of the cultured plasmid as well as comparison with positive clones (Ji et al., 2007; Kang et al., 2007) (Figure 1).

\section{Transfection with lentivirus}

GSCs were divided into an experiment group, a control group, and a blank group and subjected to CPEB interference, transfection with empty vector, and normal culture, respectively. All cells were inoculated on 6 -well plates 1 day before transfection and cultured at room temperature. When cell confluence exceeded $30 \%$, serum-free culture medium was used. When the multiplicity of infection reached 15, lentiviral vector and $5 \mu \mathrm{g} / \mathrm{mL}$ transfection enhancer (Pomerantz et al., 1990; Equils et al., 2006) were added to each well of the experiment group. Empty vector was added to each well of the control group. 


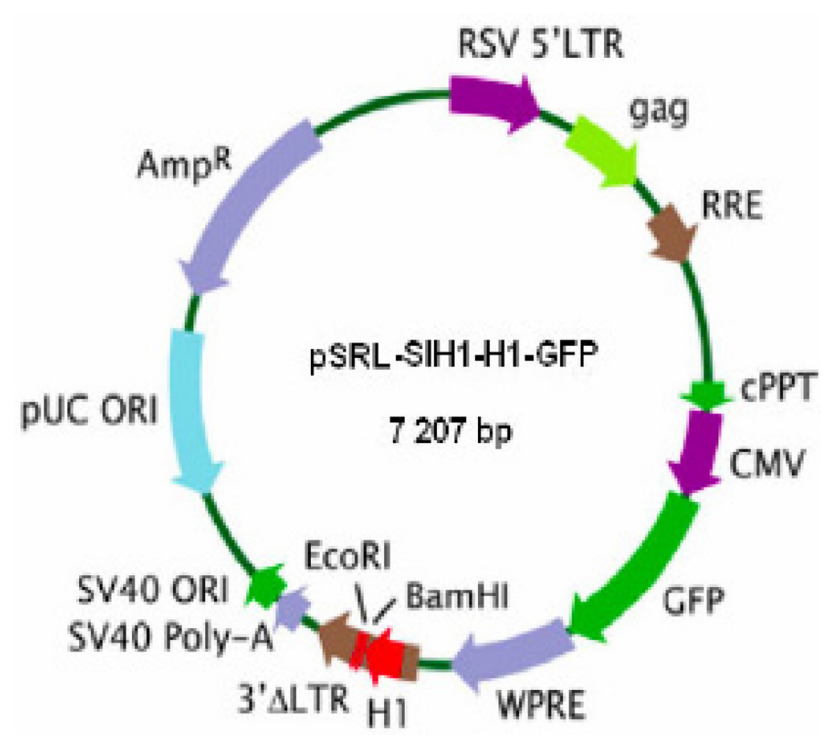

Figure 1. Constitution of lentiviral vector (including pSRL-SIH1-H1-GFP, pHelper 1.0, and pHelper 3.0 plasmids).

\section{Observation indices}

\section{Detection of CPEB expressions}

Total DNA and total proteins of each group were extracted 24, 48, 72, 96, and $120 \mathrm{~h}$ after treatment and reverse-transcribed. Expression changes in mRNA and proteins were detected by western blotting (Hansen et al., 2009). Cell cycle was detected by flow cytometry to observe proliferation. Cell viability was detected using an MTT assay.

\section{Detection of invasion ability}

Cell invasion ability was determined using a transwell assay (Li et al., 2013) in triplicate. Invading cells were counted under 8 randomly selected visual fields (200X).

\section{Statistical analysis}

All data were analyzed using SPSS 13.0 (SPSS, Inc., Chicago, IL, USA). The numerical data were compared by $\mathrm{X}^{2}$ test, and the categorical data were compared by the $t$-test. $\mathrm{P}<0.05$ was considered to be statistically significant.

\section{RESULTS}

\section{Effects of siRNA interference}

The primer sequences and interference outcomes are summarized in Table 1. Western blotting showed that siRNA-3 had the best interfering effect on CPEBs (Figure 2). 
Table 1. siRNA primer sequences and interference outcomes.

\begin{tabular}{lclr}
\hline No. & Starting point & DNA sequence & Relative expression (means \pm SD) \\
\hline siRNA-1 & 1436 & GCAAGCAATACTGGGAAT & $0.581 \pm 0.039$ \\
siRNA-2 & 1716 & GCTGGAATACTGCCTGAAA & $0.312 \pm 0.041$ \\
siRNA-3 & 1807 & GGATCGAATCTCCAGTGTT & $0.070 \pm 0.009$ \\
Control & - & 5'-AATTCTCCGAACGTGTCACGT-3' & - \\
\hline
\end{tabular}
Control
$s i \mathbf{R}-1$
S i $R=2$
$\mathrm{si} \mathbf{R}-\mathbf{3}$

CPEB 4

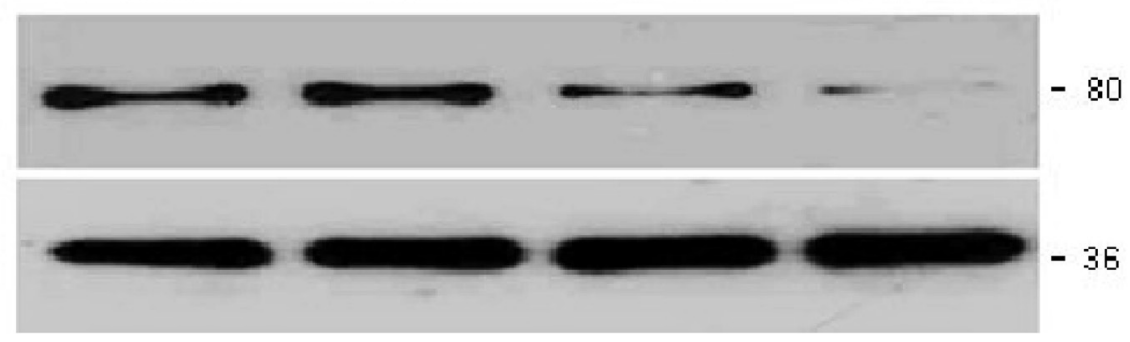

Figure 2. siRNA interference outcomes detected by western blotting.

\section{Transfection outcomes}

CPEBs were expressed in the experiment group with green fluorescence at an expression rate of over $70 \%$ (Figure 3 ).
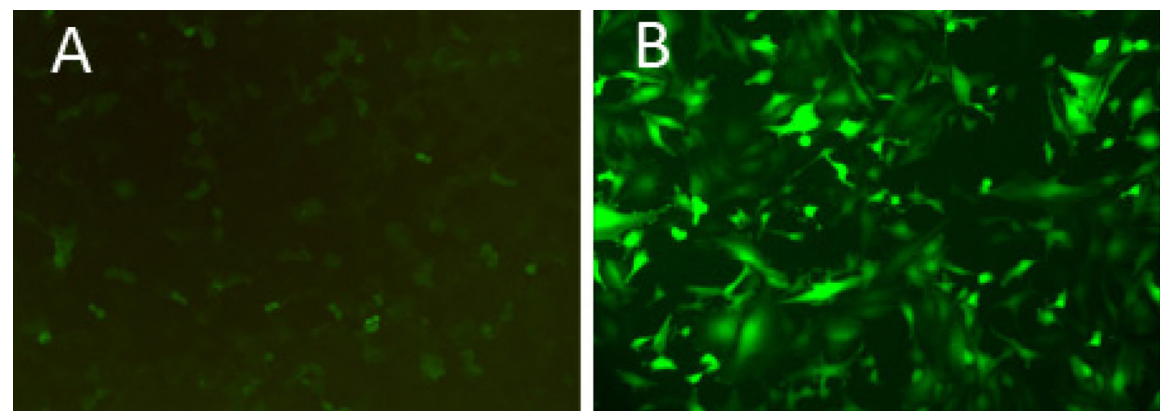

Figure 3. Green fluorescence of blank group (A) and experiment group (B) (200X).

\section{Expressions of CPEBs}

Significantly lower CPEB levels were observed in the experiment group compared to in the control and blank groups $(P<0.05)$ (Table 2).

Table 2. CPEBs expression (means $\pm \mathrm{SD}$ ).

\begin{tabular}{llll}
\hline Group & $24 \mathrm{~h}$ & $48 \mathrm{~h}$ & $72 \mathrm{~h}$ \\
\hline Experiment & $0.589 \pm 0.102$ & $0.196 \pm 0.084$ & $0.085 \pm 0.014$ \\
Control & $0.823 \pm 0.114^{*}$ & $0.819 \pm 0.118^{*}$ & $0.820 \pm 0.121^{*}$ \\
Blank & $0.858 \pm 0.129^{*}$ & $0.843 \pm 0.104^{*}$ & $0.844 \pm 0.117^{*}$ \\
\hline
\end{tabular}

${ }^{*}$ Compared with experiment group, $\mathrm{P}<0.05$. 


\section{Cell apoptotic rates}

After 48-h treatment, the apoptotic rate of the experiment group was $21.43 \%$, which was significantly higher than in the blank $(0.51 \%)$ and control $(1.43 \%)$ groups $(P<0.05)$ (Figure 4$)$.
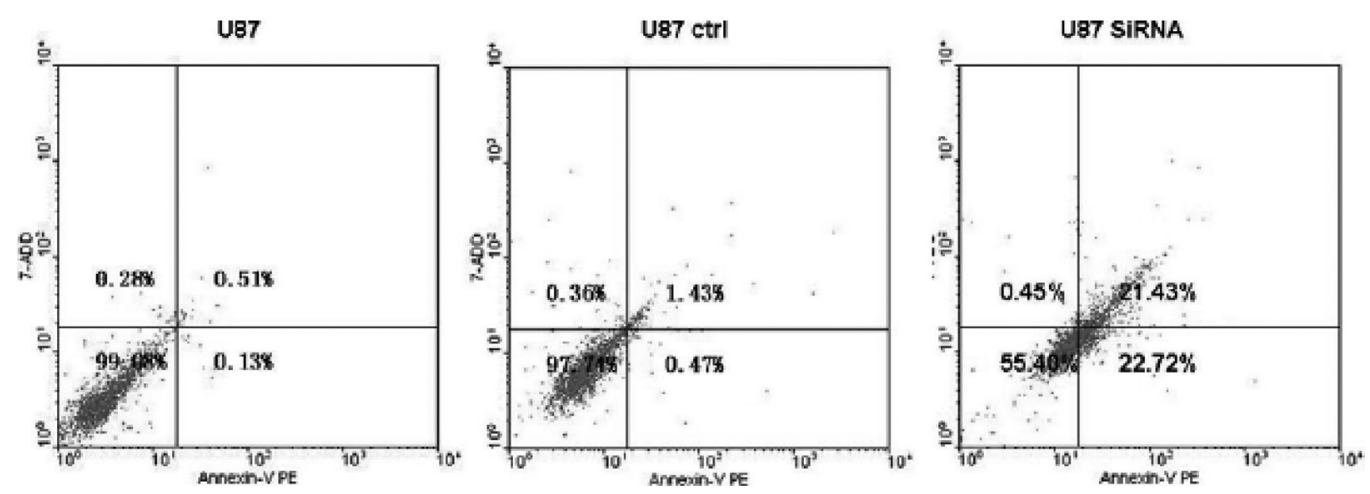

Figure 4. Cell apoptosis rates.

\section{Cell viability}

After 3 days of treatment, the experiment group grew significantly more slowly than control and blank groups $(\mathrm{P}<0.05)$ (Table 3$)$.

Table 3. Cell viability (means \pm SD).
\begin{tabular}{lcccc}
\hline Group & 1 day & 2 days & 3 days & 4 days \\
\hline Experiment & $0.397 \pm 0.025$ & $0.391 \pm 0.038$ & $0.415 \pm 0.049$ & $0.426 \pm 0.038$ \\
Control & $0.385 \pm 0.026$ & $0.402 \pm 0.065$ & $0.481 \pm 0.052^{*}$ & $0.591 \pm 0.042^{*}$ \\
Blank & $0.390 \pm 0.031$ & $0.410 \pm 0.046$ & $0.490 \pm 0.048^{*}$ & $0.609 \pm 0.035^{*}$ \\
\hline
\end{tabular}

*Compared with experiment group, $\mathrm{P}<0.05$.

\section{Cell invasion ability}

The transwell invasion assay showed that significantly fewer cells in the experiment group penetrated the membrane than those in control and blank groups did $(P<0.05)$ (Table 4).

\section{Table 4. Cell invasion ability (means \pm SD).}

\begin{tabular}{lc}
\hline Group & Number of membrane-penetrating cells (per visual field) \\
\hline Experiment & $49.32 \pm 6.97$ \\
Control & $153.61 \pm 11.84^{*}$ \\
Blank & $201.43 \pm 19.33^{*}$ \\
\hline
\end{tabular}

${ }^{*}$ Compared with experiment group, $\mathrm{P}<0.05$.

\section{DISCUSSION}

As one of the most common tumors in the human body, glioma has been treated clinically using many methods, but there are no effective protocols for improving the prognosis and quality 
of life (Dong and Huang, 2011). Therefore, studies have focused on the pathogenesis and targeted therapy of glioma (Ahmed et al., 2013). CPEBs are highly expressed in many types of malignant tumors and associated with study and memory, indicating that they may be involved in inherited neurological disorders in addition to the mediation of stem cell development, cell differentiation, aging, and synaptic plasticity (Bishnoi et al., 2008; Fernández-Miranda and Méndez, 2012).

Glioma affects the prognosis of patients mainly because of strong invasion and infiltration, and thus inhibiting the migration of GSCs using non-surgical methods is of great significance (Das et al., 2008). CPEBs are highly conservative RNA binding proteins that predominantly induce the translation of polyadenylation. Because overexpressed CPEBs remarkably promote the growth, development, and angiogenesis of tumor cells (Hansen et al., 2009; D'Ambrogio et al., 2013), we inhibited CPEBs using siRNA-3 for transfection. Characterized by a wide range of host and regular expressions of exogenous genes, lentivirus has been extensively used in gene therapy (Lee et al., 2013). In this study, transfection with a CPEB RNA-interfering lentiviral vector generated stable siRNA, and more than $70 \%$ of cells in the experiment group emitted green fluorescence, suggesting that the lentivirus carrying the specific target interference sequence worked well after transfection. With an extended transfection time, the experiment group showed dramatically decreased in CPEB expression, cell viability and invasion ability as well as an increased cell apoptotic rate. Thus, CPEB interference effectively suppressed the growth and proliferation of GSCs, showing promise for the clinical treatment of glioma. Biological functions were altered with decreasing CPEB expressions. Inhibition of mRNA translation was weakened. By forming cytoplasmic polyadenylation ribonucleoprotein complexes or by binding the Maskin protein, highly-expressed CPEBs maintain the poly A tail of mRNA to be short and make recognition of the start codon by $40 \mathrm{~S}$ ribosomal subunit difficult. As a result, translational output from mRNA cannot be continued (D'Ambrogio et al., 2013). Activation of mRNA translation is attenuated. In contrast, CPEBs extend the poly $A$ tail and the length of $m R N A$, facilitating the activation and translation of dormant mRNA, generating the cell signaling cascade, and inducing abnormal mRNA translation. Furthermore, CPEBs significantly control aggravating cancerization by playing crucial roles in cell aging and synaptic plasticity (Richter, 2007; Burns and Richter, 2008), and thus are important in the onset, proliferation, invasion, and metastasis of glioma. Hence, it is feasible that controlling CPEBs is of great significance for glioma treatment.

After CPEB interference, the growth, proliferation, and invasion of GSCs were considerably suppressed, providing valuable support for targeted therapy of glioma and for improving prognosis.

\section{Conflicts of interest}

The authors declare no conflict of interest.

\section{REFERENCES}

Ahmed AU, Auffinger B and Lesniak MS (2013). Understanding glioma stem cells: rationale, clinical relevance and therapeutic strategies. Expert Rev. Neurother. 13: 545-555.

Bishnoi M, Chopra K and Kulkarni SK (2008). Differential striatal levels of TNF-alpha, NFkappaB p65 subunit and dopamine with chronic typical and atypical neuroleptic treatment: role in orofacial dyskinesia. Prog. Neuropsychopharmacol. Biol. Psychiatry 32: 1473-1478.

Burns DM and Richter JD (2008). CPEB regulation of human cellular senescence, energy metabolism, and p53 mRNA translation. Genes Dev. 22: 3449-3460.

D'Ambrogio A, Nagaoka K and Richter JD (2013). Translational control of cell growth and malignancy by the CPEBs. Nat. Rev. Cancer 13: 283-290. 
Das S, Srikanth M and Kessler JA (2008). Cancer stem cells and glioma. Nat. Clin. Pract. Neurol. 2008. 4: 427-435.

Dong J and Huang Q (2011). Targeting glioma stem cells: enough to terminate gliomagenesis? Chin. Med. J. 124: 2756-2763. Equils O, Salehi KK, Comataeanu R, Lu D, et al. (2006). Repeated lipopolysaccharide (LPS) exposure inhibits HIV replication in primary human macrophages. Microbes Infect. 8: 2469-2476.

Fernández-Miranda G and Méndez R (2012). The CPEB-family of proteins, translational control in senescence and cancer. Ageing Res. Rev. 11: 460-472.

Hansen CN, Ketabi Z, Rosenstieme MW, Palle C, et al. (2009). Expression of CPEB, GAPDH and U6snRNA in cervical and ovarian tissue during cancer development. APMIS 117: 53-59.

Ji Y, Nie B, Li P, Xu X, et al. (2007). Construction of Hsp90beta gene specific silencing plasmid and its transfection efficiency. Front. Med. China 1: 253-257.

Kang Y, Liao WM, Sheng PY, Zhang LJ, et al. (2007). [Construction and identification of human bone morphogenetic protein-7 recombinant adeno-associated virus type 2 vector and its expression in bone mesenchymal stem cells]. Zhonghua Wai Ke Za Zhi 45: 1279-1283.

Lee HK, Bier A, Cazacu S, Finniss S, et al. (2013). MicroRNA-145 is downregulated in glial tumors and regulates glioma cell migration by targeting connective tissue growth factor. PLoS One 8: e54652.

Li P, Zhou C, Xu L and Xiao H (2013). Hypoxia enhances stemness of cancer stem cells in glioblastoma: an in vitro study. Int. J. Med. Sci. 10: 399-407.

Pomerantz RJ, Feinberg MB, Trono D and Baltimore D (1990). Lipopolysaccharide is a potent monocyte/macrophage-specific stimulator of human immunodeficiency virus type 1 expression. J. Exp. Med. 172: 253-261.

Richter JD (2007). CPEB: a life in translation. Trends Biochem. Sci. 32: 279-285.

Xiao L, Cheng J, Guo J, Zhang LY, et al. (2008). Screening and cloning genes transactivated by TGF beta 1 in hepatic stellate cells using suppression subtractive hybridization technique. Zhonghua Gan Zang Bing Za Zhi 16: 854-857. 